\title{
Strates
}

STRATES Matériaux pour la recherche en sciences sociales

9 | 1997

Crises et mutations des territoires

\section{Les « exclus » : minorités de droit ou communautés de fait}

Béatrice Mesini

\section{(2) OpenEdition}

1 Journals

Édition électronique

URL : http://journals.openedition.org/strates/614

DOI : $10.4000 /$ strates.614

ISSN : $1777-5442$

Éditeur

Laboratoire Ladyss

Édition imprimée

Date de publication : 30 septembre 1997

ISSN : 0768-8067

Référence électronique

Béatrice Mesini, «Les « exclus » : minorités de droit ou communautés de fait », Strates [En ligne], 9 ।

1997, mis en ligne le 19 octobre 2005, consulté le 08 septembre 2020. URL : http://

journals.openedition.org/strates/614; DOI : https://doi.org/10.4000/strates.614

Ce document a été généré automatiquement le 8 septembre 2020

Tous droits réservés 


\title{
Les « exclus » : minorités de droit ou communautés de fait
}

\author{
Béatrice Mesini
}

«Quelle est la source de ce souci prématuré d'établir des lois fondamentales ? C'est la vieille vanité qui conduit à se prétendre plus sage que la postérité lointaine - plus sage que ceux qui auront eu plus d'expérience ; le vieux désir de régner sur la postérité ; la vieille recette pour mettre les morts à même d'enchaîner les vivants. » J. Bentham

1 Erigés en groupe-phare dans les discours politiques, médiatiques et scientifiques depuis la deuxième moitié des années quatre-vingt, les « exclus » se constituent-ils en groupe social, telle est la question qui organise mes recherches en cours. Une place importante est accordée ici à l'analyse de la presse, où s'expriment leurs revendications d'intégration et leur refus de l'éviction: d'être sans ressources, sans emploi, sans logement, sans famille, non Français, hors-statuts, évoluant dans une «zone de nondroit ».

2 C'est à partir des commentaires, des articles, des témoignages et des points de vue recueillis, d'octobre 1994 à décembre 1996, dans les quotidiens Libération et Le Monde, le mensuel Le Monde diplomatique, que, dans cet article, je tente de cerner les formes et le sens de l'action collective au nom de l'exclusion.

Aux frontons d'une mobilisation, la garantie des principes constitutionnels

3 Les journaux nationaux sont très diversement impliqués dans le suivi d'un mouvement multisectoriel, en lutte contre les effets pervers de la crise. D'une part, l'effet de surenchère, produit par la centralité des porte-parole autorisés, conduit à dissoudre le fond commun des revendications dans la singularité des engagements de chacun. D'autre part, l'information des faits de résistance est plus ou moins rapide et dense 
suivant la ligne des quotidiens archivés ${ }^{1}$. Enfin, la nature des enjeux est peu confrontée à la complexité théorique et à l'inflation tendancielle du dispositif législatif.

Les dynamiques de l'action collective

4 Le corpus de textes qui étaye cette analyse positionne, six mois avant les campagnes électorales de 1995, un vaste forum sur l'exclusion qui met face à face les revendications de la société civile et les propositions de programme des partis politiques. L'hétérogénéité des représentations du groupe des "exclus" évolue dialectiquement sous l'effet d'attraction qu'il suscite dans les espaces de légitimation qui s'affrontent autour de revendications multiples. Les artistes, enseignants et scientifiques se sont tour à tour investis dans la défense du libre accès aux droits de la personne, soutenus dans leurs actions par le mouvement international de lutte contre la pauvreté et l'exclusion, et relayés par voie de presse.

5 La fusion des revendications, en préparation dès le mois d'octobre 1994 lors de la journée mondiale du refus de la misère et de la Journée nationale de la solidarité se déploie, le 6 mars 1995, au sommet de Copenhague. Avec 183 pays représentés, 200 organisations non-gouvernementales, et un contre-sommet regroupant 700 O.N.G., le rassemblement déclare la guerre à la pauvreté et l'exclusion. Dans son discours d'ouverture, le secrétaire général des Nations-Unies Boutros Boutros-Ghali invite la communauté des nations à «réfléchir à un nouveau projet de vie collective » et évoque :

6 "l'impérieuse obligation d'un nouveau contrat social pour offrir aux nations, aux hommes et aux femmes du monde des raisons d'espérer [...]. En élevant la question sociale au rang des priorités universelles, nous voulons, par là-même, prendre en charge le devenir collectif de la société internationale et conclure un nouveau pacte de solidarité à l'échelle de la planète [...]. Pour faire face à cette attente et pour mettre en ceuvre une véritable politique internationale du développement social, trois grandes préoccupations doivent inspirer nos débats: la redistribution, l'intégration, la protection [...]. Il nous faudra garder présent à l'esprit que le meilleur facteur d'intégration reste, pour l'individu, le sentiment d'appartenancé2."

7 Le mouvement revendicatif s'exacerbe le 8 avril 1995 en France avec la manifestation unitaire "Pour les droits et l'égalité, Contre les exclusions et la précarité ». Une centaine d'associations et d'organisations, se définissant comme « un contre-pouvoir de la société civile ", défilent, soutenues par 20000 personnes pour " exercer une pression sur la classe politique et sur les candidats présidentiels ", en les interpellant sur le sida, le chômage, le logement, le racisme. Sont présents le D.A.L. (Droit au logement), les associations de mal-logés, le M.R.A.P.(Mouvement contre le racisme et pour l'amitié entre les peuples), S.O.S. racisme, le F.A.S.T.I.(Fédération des associations de solidarité avec les travailleurs immigrés), la C.G.T., la C.F.D.T., A.I.D.E.S...

8 La mobilisation unitaire est renforcée par l'implication des autorités spirituelles de plusieurs confessions ${ }^{3}$. Le 12 avril 1995, un appel inter-religieux est lancé à l'initiative de J.B. de Foucauld ${ }^{4}$ :

9 "Il y a le choix inavouable d'un schéma de croissance qui enrichit globalement la communauté nationale, mais qui la laisse se distendre et se disloquer. Les anciennes régulations ne suffisent plus à conjurer cette dérive: les ressources à mobiliser sont aussi, désormais, spirituelles et morales, car c'est d'abord l'indifférence qui est responsable des fractures qui se dessinent ${ }^{5}$.»

10 De même, la laïcité républicaine se réaffirme quelques jours plus tard dans les propos de P. Kessel, grand maître du Grand-Orient de France : 
11 «Il ne peut y avoir de République qui regarde avec résignation l'exclusion. [...] Il faut réaffirmer la liberté, qui n'est pas le libéralisme; réaffirmer l'égalité, qui n'est pas l'équité, notamment en matière d'éducation [...]; il faut, enfin, réaffirmer la fraternité, le mot apparemment le plus désuet de la République, mais le plus important. Les gens ont besoin de l'autre. La solidarité et la confirmation d'une protection sociale n'ont rien à voir avec la charité et elles ne peuvent donc pas être concédées au marché ${ }^{6}$ "

12 La vague des grèves qui émaille l'automne/hiver 1995, réunit côte à côte les « exclus » et les partenaires sociaux dans l'expression d'une révolte orchestrée contre les réformes et les programmes politiques octroyés sans négociations préalables :

13 "L'unité de la crise vient d'abord de ce qu'elle est liée à la légitimité du pouvoir actuel, élu non pour moderniser le pays, mais sur la base d'engagements renvoyant à la "fracture sociale" 7."

14 "Mais le mouvement ne se contente pas de mettre le pouvoir à la place du mort. Il expérimente pratiquement une manière différente de vivre, une condition sociale qui pourrait bien fonctionner en service minimum, et capable de déployer une énergie fantastique en l'absence d'État et de système de contrôle [...]. C'est une fracture non seulement sociale mais mentale [...]. Deux forces antagonistes qui ne relèvent plus de la lutte de classes: l'une, la puissance rationnelle, maîtresse des signes et du langage [de moins en moins], des techniques de persuasion et de discussion [de plus en plus] : l'autre, fragmentaire, erratique, non représentative, celle à qui l'on a imposé le sens linéaire du progrès et de l'Histoire. Cette puissance-là, personne ne veut la reconnaître. Car personne ne comprend les préparatifs souterrains de la colère ${ }^{8}$. "

15 Le mouvement des sans-papiers, initié en été 1996 à la suite de l'évacuation de l'église Saint-Bernard, se cristallise ; il est relayé à la base par des collectifs de sans-papiers qui organisent les actions dans les communautés africaine mais aussi chinoise, turque, haïtienne :

16 "Nous sommes allés montrer aux sénateurs de la République nos fiches de paie, nos déclarations d'impôts, notre sécurité sociale. Nous ne demandons rien, ni travail, ni logement. Nous voulons seulement sortir de l'ombre et combattre les lois qui nous ont mis là̀. "

17 La manifestation de solidarité avec les immigrés « ni expulsables, ni régularisables »a, pour sa part, réuni quinze mille personne à l'automne 1996 réclamant le «droit de vivre ici ${ }^{10}$. "

La bannière des droits universels

18 La mobilisation en faveur des droits unifie l'exigence d'un débat sur les identités collectives vécues par-delà les statuts octroyés de chômeurs, Rmistes, handicapés, malades, précaires, clandestins. Si la question du droit est alors dominante, c'est parce qu'elle porte en substance l'hypothèse d'une construction ambiguë d'un groupe intégré en droit a minima et exclu, dans les faits, de la communauté nationale :

19 «Des personnes sont mises à l'écart de la société parce qu'elles sont jugées indignes ou incompétentes de jouir des droits et bénéfices accordés aux citoyens ordinaires, lesquels représentent à la fois la norme et la majorité. Une telle malédiction peut atteindre aussi bien l'étranger que "l'anormal", ou encore la "personne en difficulté »; c'est-à-dire tous ceux qui, soit par leur origine géographique ou ethnique, soit par leur handicap génétique ou acquis, soit encore par leur comportement ou leur malchance, ont dévoilé une différence insupportable avec le modèle majoritaire ${ }^{11}{ }^{\prime}$.

20 Science de normes, le droit ne s'élabore qu'à partir de considérations psychologiques, éthiques et politiques, étrangères et souvent supplétives à la science juridique ellemême - usages, coutumes - ; les considérations politiques pèsent sur les préceptes 
démocratiques à l'aune des connaissances, des savoirs et des nécessités de chaque époque. Les Constitutions successives n'ont fait que retranscrire l'évolution conflictuelle entre droits créances et droits devoirs; la déclaration des droits de l'homme de 1793 (non appliquée), la déclaration des droits et des devoirs de l'homme et du citoyen de l'an III, la Constitution du 4 novembre 1848, le préambule de la déclaration des droits de l'homme du 19 avril 1946 (rejetant la clause de relativité de la propriété) et le préambule de la Constitution de 1958 font tous référence à cette déclaration des droits qui fixent les principes fondamentaux obligatoires.

Or l'exclusion érode et fragmente le socle des droits de la première génération de 1789 : l'égalité des citoyens, le droit de la propriété - quand les réquisitions en dissocient les fondements (usus, abusus, fructus) - ainsi que le droit de la famille tandis que se recomposent les filiations - projet de contrat d'union civile, certificat municipal de concubinage pour les couples homosexuels, projet de contrat d'union sociale... Les droits sociaux, droits collectifs de la deuxième génération consacrés après-guerre, sont pour leur part invalidés dans l'affirmation du droit d'obtenir un emploi et des moyens convenables d'existence (alinéas 4 et 11, préambule de la Constitution du 27 octobre 1946).

La reconduction des droits de 1789 dans la constitution de $1958^{12}$ ne saurait masquer l'essentiel: s'ils sont des textes "sacrés", régulièrement invoqués devant la Cour européenne des droits de l'homme ${ }^{13}$ pour soutenir l'individu résistant contre les lois nationales, ils sont toutefois dépouillés de leur valeur intrinsèque : la garantie de leur application dans le fonctionnement de la démocratie :

23 "Car, si aux 3,5 millions de sans-emploi, on ajoute les exclus de toutes sortes, cela fait une population de 6 millions de paupérisés [...]. Parce que la misère est une insulte aux droits de l'homme, de telles déchirures dans le tissu social ruinent une certaine conception de la République. Les chômeurs, les sans-abri, les Rmistes, les précarisés, les exclus sont l'expression dramatique des sacrifices réclamés sans contrepartie, à la société française depuis deux décennies. La traduction sociale de choix purement idéologiques, fondés sur la rigueur budgétaire, les délocalisations, la compétitivité, la productivité, etc. De cela, les gens ne veulent plus. Ils n'acceptent pas qu'on appelle "réforme" ce qui n'est, au sens propre, qu'une contreréforme, un retour à l'ordre social ancien. ${ }^{14}$ "

$24 \mathrm{Au}$ centre des questions d'exclusion se délite le cadre protecteur des droits fondamentaux de l'homme quand se légitiment chaque fois davantage les violations légales et les distorsions aux préceptes de liberté, d'égalité et de fraternité : négation du droit au travail, conditionnalité du droit à la subsistance, du droit du sol, principes de discrimination positive entre les territoires et les individus, déni du droit d'asile... En deux siècles, la question des droits fondamentaux a évolué du sentiment passionné pour la liberté politique à celui d'une régression juridique - inflation de statuts dérogatoires au droit commun - négociée par les gouvernements successifs, au nom d'impératifs économiques dictés par la crise et la compétitivité mondiale.

En guise de contrat politique : la défense des droits sociaux

25 Tiraillé entre l'universalisme et le communautarisme, l'État programme la fiction anachronique d'un modèle national d'intégration, scandé par les termes de groupescibles, de territoires ségrégés, de cultures minoritaires.

La relégitimation permanente de l'action publique

26 La gestion politique $d u$ "changement» polarise, dans un premier temps, les représentations de l'exclusion sur le double primat social/spatial des populations 
désœuvrées et des territoires "à risque $»^{15}$ qu'il faut insérer. Figures collectives régulièrement exhumées par la presse, hordes, tribus, bandes, gangs, ethnies et communautés peuplent des territoires « en sécession » et constituent des minorités qui menacent la cohésion du pacte national : "Le besoin de sécurité est apparu comme l'un des principaux signaux du premier tour de scrutin [...]. Comment tolérer que des cités entières, des quartiers en sécession échappent au droit ? ${ }^{16}{ }^{\prime}$.

Les échéances présidentielle et municipale de 1995 enregistrent, au travers des sondages réalisés durant cette période, une sensibilité accrue de la population au fait de l'exclusion: $80 \%$ se déclarent favorables à l'application de la loi de réquisition des logements, $85 \%$ sont pour la gratuité des transports pour les chômeurs, $82 \%$ pour l'extension à tous de la sécurité sociale, $74 \%$ souhaitent l'extension du R.M.I. aux 18/25 ans ${ }^{17}$.

Les candidats à la présidentielle ont chacun investi le créneau en proposant des platesformes, autour de la citoyenneté, susceptibles de répondre aux préoccupations de leurs électeurs: plan Marshall sous tutelle de l'État à base de fiscalité dérogatoire et de déconcentration, pour J. Chirac ; procédure contractuelle de la République sociale pour L. Jospin, qui prône la liberté par la citoyenneté, l'égalité pour la justice et la fraternité dans la paix ; mise en œuvre du devoir de fraternité et réformes inspirées par l'équité pour E. Balladur ; politique de transformation sociale pour D. Voynet... La lutte contre l'exclusion est proclamée comme priorité des maires: en juin 1995, 74\% des municipalités de plus de 30000 habitants ont passé un contrat de ville avec l'État, tandis que les deux tiers des plans locaux d'insertion par l'économique, mis en place dans $50 \%$ de villes, sont opérationnels.

30 La définition, sur le terrain, des propositions électorales conduit inexorablement à la mise en concurrence des initiatives et des priorités entre les compétences décentralisées des territoires. Le pacte de relance pour la ville a pour objectif de favoriser l'intégration urbaine par l'emploi, grâce à une politique de défiscalisation et d'aides en crédits dans des zones délimitées ; présenté à Marseille, le 18 janvier 1996, par A. Juppé, il offre une large palette de mesures économiques, sociales et sécuritaires qui touchent à tous les aspects de la vie dans les sept cents zones sensibles répertoriées : création de trois cent cinquante zones de revitalisation urbaine, de trente huit zones franches, de 100000 emplois de ville pour les jeunes, supplément de quatre mille policiers en trois ans, traitement spécial de la délinquance des mineurs, aménagement de sites urbains prioritaires pour l'éducation, réhabilitation de cinq cent mille logements, crédits d'équipement en direction des communes pour le développement social urbain...

31 Ces mesures se doublent dans la foulée d'un plan de sauvetage des campagnes: le conseil national de l'aménagement adopte, le 5 février 1996, une série de dispositions pour redynamiser les territoires fragilisés par la désertification et le chômage :

32 "Ces zones de revitalisation rurale comprennent les arrondissements, les cantons, les communes dont la densité ne cesse de fléchir et qui atteignent des seuils alarmants: 33 habitants au kilomètre carré pour les arrondissements, 31 pour les cantons, 5 pour les communes. Cette définition permet de calculer que près de $40 \%$ du territoire national correspond dorénavant à cette entité administrative. Et couvre plus de quatre millions et demi de français ${ }^{18}$." 

clairement que, en défendant d'abord leurs droits, ils ont aussi défendu ceux des autres en posant des questions plus générales sur l'évolution de notre société et les choix socioéconomiques de nos gouvernants ${ }^{24} "$; destruction d'une "civilisation" associée à l'existence du service public, celle de l'égalité républicaine des droits, droit à l'éducation, à la santé, à la culture, à la recherche, à l'art, et, par dessus tout, au travail ${ }^{25}$."

41 En discussion depuis octobre 1995, le projet de loi contre l'exclusion prévoyait, dans le programme du candidat J. Chirac, une refonte générale du dispositif de lutte contre la pauvreté. Cette réforme chiffrée à plus de quarante milliards de francs propose l'élaboration d'une « loi d'orientation qui, dans tous les domaines de la vie politique, économique, sociale et culturelle, scelle solennellement l'engagement de la Nation toute entière pour vaincre l'exclusion ${ }^{26} »$. Promise lors des vœux de fin d'année du 
président pour le printemps 1996, la discussion sur la loi-cadre reprogrammée à la session d'automne du parlement sera débattue au Parlement au premier trimestre 1997.

L'avant-projet de loi dévoilé le 30 septembre 1996 cautérise l'écart entre la garantie formelle des droits et leur exercice dans la cité : "j'attends de cette loi contre l'exclusion qu'elle conforte ces droits fondamentaux et les traduisent dans les faits ${ }^{27}$. Le «programme d'action pour le renforcement de la cohésion sociale " s'organise autour de cinq grands objectifs :

43 - garantir la dignité en matière de citoyenneté (droit de vote des SDF, droit de vivre en famille, mise en place d'un médiateur dans les organismes de protection sociale) ;

- transformer les allocations sociales en salaires en modifiant les « dépenses passives en dépenses actives »;

45 - développer la prévention en matière de santé (tuberculose, saturnisme et généralisation de l'accueil social à tous les hôpitaux);

46 - faciliter les réquisitions (qui s'appliqueront aux personnes morales et aux logements inoccupés depuis 18 mois), éviter les expulsions;

47 - donner la priorité nationale à la lutte contre l'illettrisme.

Ces propositions mettent l'accent sur la nature des droits proclamés - citoyenneté, emploi, santé, logement, éducation - et sur les garanties légales assurant leur accès à tous les individus : « la loi garantit l'accès aux droits fondamentaux dans le domaine des droits individuels (...), des droits collectifs et sociaux ainsi que dans les domaines privilégiés de l'intégration sociale ${ }^{28}$."

Les droits politiques au fondement de la participation

49 Couronnée durant la campagne présidentielle, popularisée depuis dans les discours politiques, médiatiques et scientifiques, l'expression de « fracture sociale » entérine un triple choix idéologique du gouvernement: atomiser les revendications dans un contexte d'urgence de "demande sociale », en empilant les dispositifs de replâtrage ; inscrire les transgressions au droit commun dans la mise en œuvre d'un contrôle renforcé (des chômeurs, des Rmistes et de leur famille, des concubins, des mendiants, des associations ; mais aussi des quartiers, des banlieues, des zones urbaines, rurales, et des frontières); pérenniser un modèle de développement économique qui met en exergue les exclus face aux classes moyennes, pour gérer le déclin des statuts liés à l'emploi.

Devoirs d'intégration ou droits d'appartenance

50 Les statuts juridiques d'appartenance au travail, à la famille et au territoire sont invalidés quotidiennement par les indicateurs quantitatifs: plus de 3 millions de chômeurs, 1,8 million de personnes avec conjoints et enfants bénéficiaires du R.M.I., 202000 exclus du logement dont 98000 sans domicile fixe, 1,2 million de familles monoparentales, 500000 familles en difficulté face au fisc... Ces indicateurs approximatifs renvoient, non pas à l'histoire officielle d'une participation révolue au destin de la communauté nationale mais à l'histoire de ses appartenances communautaires (en creux du droit commun), dans et par l'expérience de modes de vie solidaires.

51 Inscrivant l'individu dans des «nous» inclusifs, les appartenances multiples - artistique, culturelle, spirituelle, philosophique, ésotérique, émotionnelle - activent 
une mémoire créatrice et réflexive de l'être au monde, d'« appels à être » de l'identité, contre l'absolutisme de la domination économique et juridique :

"Prenons la liste des associations qui se créent. Selon les intitulés qu'elles se donnent, on peut distinguer des associations de commodité - faire ensemble - et des associations de communauté - être ensemble. Certaines associations réunissent des adhérents qui ont pour objectif de vivre des choses ensemble. D'autres se donnent pour ambition de faire des choses ensemble. Le maître mot est "ensemble". Est-ce un hasard si le slogan qui s'est imposé lors du récent mouvement social en France est : "Tous ensemble! Oui !"29."

C'est dans le renouvellement et la permanence de micro-groupes qui font lien au sein de la société que se dessine l'hypothèse d'une communautarisation en positif des appartenances :

54 "Qui sommes-nous?" ne se laisse pas confondre avec "Que sommes-nous". Ce n'est pas la question théorique de la nature humaine, mais la demande de la reconnaissance humaine. Cette question n'est pas scientifique mais politique. Elle n'a donc pas de terrain particulier car elle les a tous : la reconnaissance de l'utilité d'un travail, la reconnaissance sociale d'un statut politique, d'une souveraineté culturelle, d'une langue, d'un culte ou de mœurs [...]. "Sommes-nous" est la question qui exige l'égalité [...]. Derrière toutes les paroles humanistes, voire humanitaires, sur l'universalisme moral, elle rappelle que dans "vivre ensemble", il y a "vivre" et que la communauté ne peut durer si elle entrave le bonheur ${ }^{30}$."

55 L'efficacité du concept d'exclusion renvoie à l'idéologie parce que les représentations qui s'affrontent autour des exclus impriment une tension entre le socle juridique des droits et celui des devoirs des citoyens dans un État qui pérennise des affiliations caduques. Ce qui fait sens au centre des mobilisations, c'est la capacité de résistance collective face au futur programmé par la mondialisation :

56 "Dans cette phase historique de rupture majeure entre l'échange des hommes et l'échange des choses [...], ces "groupes sujets" se donnent des règles d'art de vivre le temps, l'espace, l'échange, la monnaie, la consommation [...].Les systèmes d'échanges locaux s'efforcent de réinventer des capacités concrètes d'échanges accessibles à tous, y compris pour les exclus de la spéculation régnante qui ne leur laisse le choix qu'entre la résignation à la misère ou le refuge dans l'assistance. Au lieu d'attendre "ce qui va arriver", ils tentent dès aujourd'hui de résister à la barbarie, c'est-à-dire à une espèce où il n'y a ni foi, ni loi ${ }^{31}$. "

57 «Par leur formidable révolte sociale de décembre 1995, les Français ont collectivement, pour la première fois, exprimé leur refus d'un modèle de société fondé sur l'économisme, le libéralisme intégral, le totalitarisme des marchés et la tyrannie de la mondialisation. Ils ont rappelé aux dirigeants un vieux principe républicain : les citoyens préfèrent le désordre à l'injustice ${ }^{32}$."

De ce point de vue, la "panne des institutions intégratrices » ne se résoudra pas en saupoudrant les lois nationales d'un zeste de " différence culturelle ${ }^{33}$, mais bel et bien en interrogeant le sens et l'essence de modes pluriels d'appartenance :

59 "L'essentiel des modifications dans nos comportements, nous les avons importés, acceptés, voulus, d'Inde, du Maghreb, d'Afrique, d'Asie et pas seulement des États-Unis. Nous nous déclarons et nous nous voulons frères et sœurs de tous [...]. Où sont-elles toutes ces valeurs que vous nous avez enseignées? C'est pour ce monde là, pour cette société-là que vous nous avez enfantés? Nous ne l'acceptons pas et nous ne l'accepterons jamais, ce monde de demain que vous voulez nous imposer. Nous sommes en guerre contre vous, contre une certaine idée de la France que vous salissez et pour une certaine idée du monde que nous chérissons ${ }^{34}$. »

La participation en acte 
60 En 1789, la déclaration inscrite en introduction à la Constitution donne au texte la forme d'un pacte s'imposant aux gouvernants ${ }^{35}$; elle fait appel à la notion de droits " naturels », inhérents aux individus en raison de leur qualité d'être humain. Les droits de l'homme consacrés dans la première déclaration des droits servent la défense de l'individu contre les lois jugées iniques; ils octroient aux citoyens des garanties fondamentales encadrant a priori la fonction législative et les protégeant dans l'exercice de leurs droits. Ainsi, «toute société dans laquelle la garantie des droits n'est pas assurée, ni la séparation des pouvoirs déterminée, n'a pas de constitution. » (article 16 de la Déclaration du 24 août 1789).

61 La question des fondements du droit apparaît décisive dans la mesure où la légitimité du pacte politique a pour corollaire la relativité des formes qu'il institue. A la différence d'une forme juridique ossifiée, la constitution d'un État de droit devrait être un principe d'actualisation de la condition humaine :

62 «Les normes juridiques deviennent ce que les homme en font [...], le droit évolue tout autant par réinterprétation de règles anciennes qu'abrogation de normes obsolètes ${ }^{36}$. "

63 Et si l'autorité du juge se requiert lorsqu'il s'agit d'énoncer l'expression des droits, elle devient suspecte lorsqu'il lui incombe, en dernier recours, de "les faire parler $\|^{37}$ :

64 "Loin de prendre acte de l'intelligence et du courage du mouvement des sans-papiers [...], le gouvernement répond par la violence, l'incohérence, le non-respect des conventions internationales, des droits de l'homme, au mépris d'un minimum d'humanités. " "

65 L'exclusion unanimement dénoncée nous rappelle que l'acceptation d'un régime politique ne peut s'incarner dans la participation effective que si celle-ci assure et garantit à l'individu le libre exercice de ces droits :

66 "Parler de "fracture sociale" est devenu une tarte à la crème du discours politique [...]. Mais que montre la réalité têtue? Au Mans, en mars 1996, le collectif Adéquat a investi le parc à fourrage, site militaire inutilisée depuis 1990 (...) Il s'agit pour eux de ranimer des lieux vides, d'implanter des activités alternatives dans des quartiers proches des fameuses "cités" fracturées. Il s'agit de vivre d'autres rapports, moins consommateurs, à la création et à la culture [c'est-à-dire à ce qui invente des formes de représentation et de compréhension du monde]... un référé est prononcé, le 10 avril, qui décide de l'expulsion. L'huissier est passé. On attend les cars de police. Les membres d'Adéquat sont tout sauf des squatters irresponsables. Ils ont un projet civique. Nous soutenons cette action parce qu'il y va de la liberté de création et de ses enjeux civiques. Nous sommes scandalisés qu'on semble, côté pouvoir, incapable de dialogue [...]. Un tel pouvoir manifeste sa peur de tout ce qui invente des espaces où s'expérimente peut-être une autre manière, plus solidaire, d'inventer et de travailler en commun. Il avoue que ses appels rituels à conjurer la fameuse "fracture sociale" ne sont que des effets de manche pour tribune électorale. Donc acte $e^{39}$ ".

67 La « démocratie pluraliste, fondée sur la diversité culturelle et la liberté, n'est pas dépassable » :

68 "Nous ne voulons plus d'une démocratie de participation; nous ne pouvons pas nous contenter d'une démocratie de délibération; nous avons besoin d'une démocratie de libération $"{ }^{40}$;

La participation ne se décrète ni politiquement dans une citoyenneté mécanique, ni institutionnellement dans des ministères de tutelle. Elle se prévaut de l'ensemble de ces actions et pratiques collectives qui retisse l'histoire d'une résistance cognitive à l'exclusion, d'une entraide entre des « nous » recomposés, d'une circulation des savoirs 
et des avoirs entre des espaces et des territoires « autonomes », libérés du marquage de la désignation :

70 "Pour l'instant les défricheurs de ces nouveaux espaces apparaissent au mieux comme les danseuses du système ou plus simplement comme de doux rêveurs [...]. Le but premier est bien d'habiter autrement la mondialisation, qui reste un espace de liberté [...]. Encore faut-il que le politique sache imaginer des médiations entre le local et le mondial. Il s'agit d'échapper à la fois au refuge sectaire dans quelques phalanstères hors du temps et à la condition "moderne" d'individus ballottés au hasard des crises monétaires d'une spéculation mondiale sans foi ni loi. ${ }^{41}$.»

71 Finalement, les exclus négocient dans le silence de l'imaginaire les dimensions cognitives d'une "rêverie sans horizons", d'un monde advenu dans la visée de ses origines $^{42}$ et dont la mémoire s'interroge :

72 «Et, de même que les sciences sociales doivent penser l'altérité et les modalités de coexistence démocratique des différences, de même l'histoire est confrontée aux défis de la démultiplication des points de vue qu'apportent les mémoires individuelles et collectives, avec leur légitimité, mais aussi leur part d'excès et de lacunes: la rigueur scientifique, le maintien d'un souci d'universalité adossé à la raison peuvent-ils encore assurer l'intégration de souvenirs et d'expériences qui plaident parfois pour de profonds changements de perspectives, et qui souvent constituent une brutale mise en cause de l'histoire établie ? ${ }^{43}$ ".

73 L'implication prudente des juristes dans l'analyse des causes et des moyens de remédier à l'exclusion nous sensibilise à la difficulté qu'il y aurait à toiletter techniquement un corpus de textes dont les principes constitutionnels se sont contredits puis invalidés au cours des temps. Faut-il «recoudre le droit $»^{44}$ ou bien le repenser dans ses fondements ${ }^{45}$ ?

74 «La France sert de référence à nombre de chercheurs et constitutionnalistes qui ont peine à sortir d'un modèle qui s'est mis en place puis épanoui sur plusieurs siècles d'histoire et qui est désormais en question: $d u$ fait de la régionalisation et de la revendication culturelle; du fait, $\grave{a}$ l'inverse, de la constitution d'ensembles régionaux auxquels les États, dont la France, confient certains de leurs pouvoirs régaliens [...]. Nous sommes en présence d'un système d'organisation globalisant. Faut-il en changer? [...] Envisageons-nous de donner une place, dans nos institutions, aux communautés, aux lieux "d'être ensemble" ? Est-ce que, à côté du concept de citoyenneté [faire], peut naître un concept d'appartenance culturelle [être] ?"46."

75 En tant que groupe réalisé dans l'imaginaire, les « exclus » ne font pas que pervertir le modèle économique d'une démocratie libérale garante en théorie des droits du citoyen; ils proposent aussi une révision concrète des statuts qui discriminent et brident aujourd'hui les appartenances vécues. Objectivée dans ses formes collectives et subjectivée par l'expérience humaine, l'identité conjugue l'abolition, l'invention et la recréation de liens en rupture avec les anciens : hors travail mais en activités ${ }^{47}$, hors mariage mais en unions libres, hors nationalité mais issus de métissages, hors domicile mais nomade, « citoyen du monde».

76 S'agit-il alors pour les «exclus » d'obtenir le droit d'avoir des droits en multipliant à l'infini les dispositifs dérogatoires au droit commun ${ }^{48}$, ou de penser l'élaboration des préceptes de ceux que l'imprescriptibilité rendent "particulièrement nécessaires à notre temps ${ }^{49} »$ ?

77 «En cette fin de siècle, il me semble que l'éthique de la communauté pourrait être élargie à tous les humains. Si je propose aujourd'hui l'idée de Terre-patrie c'est que je prône un enracinement 
plus profond dans une communauté d'origine terrienne et une conscience devenue vitale de notre communauté de destin planétaire. A cela s'ajoute une communauté de perdition, puisque nous savons que nous sommes perdus dans le grand univers, et que nous sommes tous voués à la souffrance et à la mort ${ }^{50}$."

A terme se profile une troisième génération de droits du "vivant $»^{51}$ qui concilie les droits « naturels » de l'individu et les droits « éthiques » de la personne humaine et les droits politiques du citoyen.

\section{NOTES}

1.. Ainsi les réquisitions opérées par le DAL et $\mathrm{AC}$ !, «AC! les guérilleros antichômage», Libération, 6 février 1996.

2.. Le Monde, 7 mars 1995.

3. D. Boubakeur (recteur de la mosquée de Paris), O. Clément (théologien orthodoxe),

M. Cohen (président du Consistoire de Paris), R. Draï (universitaire), Mgr A. Rouet (président de la commission sociale de l'épiscopat), D. Saada (président du Fonds social juif) et J. Stewart (président de la Fédération protestante de France).

4. Commissaire général au Plan et président de Solidarités nouvelles face au chômage.

5.. Le Monde, 13 avril 1995.

6.. Le Monde, 23/24 avril 1995.

7.. M. Wieviorka, (sociologue) « La crise est multiple et unique », Libération, 8 décembre 1995.

8.. J. Baudrillard, (sociologue), « Souveraineté de la grève », Libération, 18 décembre 1995.

9.. A. Traoré et A. Diop (porte-parole des sans-papiers), Libération, 3 octobre 1996.

10.. Le Monde, 1er octobre 1996.

11.. J. Testart (biologiste). "Puisque la fraternité, inscrite aux frontons de la République, est une qualité qui se réfêre aux semblables, son évocation contredit l'agitation de la génétique pour démontrer de nouvelles différences; pourquoi ne pas proposer que le véritable projet humaniste est d'assurer à tout citoyen "Liberté, Égalité, Fraternité" ", in Liberté, égalité, altérité, Libération, 18 juillet 1995.

12.. Via la référence au préambule de la constitution de 1946.

13.. Cf. l'affaire Spanner, à propos de la revendication de « la libre disposition de son corps ", Libération, 16 septembre 1996.

14.. I. Ramonet, « L'espoir », Le Monde diplomatique, janvier 1996.

15.. Cf. le projet des contrats de ville du XI ${ }^{\mathrm{e}}$ plan, Délégation Interministérielle à la Ville, mai 1993.

16.. J. Chirac, le 25 avril à Orléans, Le Monde, 27 avril 1995.

17.. Sondage C.S.A./La Vie, Libération, 8-9 avril 1995.

18.. Libération, 6 février 1996.

19.. Libération, 10 avril 1996. 
20.. Le R.M.I., aujourd'hui pourfendu en tant que « culture de l'oisiveté », s'élabore précisément en 1988 " sous réserve de ne pas encourager l'oisiveté » et se conditionne à la contrepartie insertion. Le rapporteur du projet de loi sur le R.M.I. énonce que « la définition d'un revenu minimal garanti repose sur des appréciations morales, idéologiques ou politiques relatives aux besoins et aux comportements des pauvres ainsi que sur l'organisation du travail et de la société marchande. " Doctrine, Actualités Juridiques, 20 octobre 1989.

21.. Dès les grèves de 1987, les chômeurs étaient utilisés par le Premier ministre J. Chirac en direction des salariés dont les revendications s'exacerbaient : "C'est dans cette direction que doit s'exercer la solidarité nationale, plus que dans celle qui consisterait à satisfaire les revendications, si légitimes soient-elles, de ceux qui se trouvent dans une situation tout de même plus favorable [...]. Le gouvernement a donc l'intention de poursuivre dans cette direction qui est celle de la rigueur, celle de la solidarité envers les plus défavorisés et celle de l'espoir. ", Le Monde, 7 janvier 1987.

22.. D. Béhar, P. Estèbe (membres du groupe ACADIE, coopérative pour la recherche et l'évaluation de la politique de la ville), « Le frisson qui rassure », Le Monde, 23 mars 1995.

23.. R. Lenoir (président de l'Union nationale interfédérale des œuvres et organismes privés, sanitaires et sociaux), Le Monde, 22-23 janvier 1995.

24.. C. Baudelot (sociologue) et S. Israël (historien), « Le bel avenir de la grève ", Le Monde, 28 décembre 1995.

25.. Intervention de P. Bourdieu (sociologue), Libération, 14 déc. 1995.

26. J. Chirac au colloque sur l'exclusion, 27 mars 1996, Libération, 28 mars 1996.

27.. J. Chirac à la journée des droits de l'homme, le 10 décembre 1996.

28. Libération, 30 septembre 1996.

29.. E. Pisani, « "Tous ensemble" contre la mondialisation », Le Monde diplomatique, janvier 1996.

30.. B. Michaux (philosophe), « "Qui sommes-nous". Quête d'un partage », Libération, 28 mars 1996.

31.. B. Ginisty (économiste), op. cit., Libération, 11-12 décembre 1995.

32.. I. Ramonet, « L'espoir », Le Monde diplomatique, janvier 1996.

33.. Les partis, les syndicats, l'école (avec une réserve pour l'Église, N.D.A.), M. Wieviorka (sociologue), Une société fragmentée, La Découverte, 1996.

34.. Les cultures, en tant que mémoire et résolution du présent dialoguent en elles et entre elles, en dépit d'une Constitution résistant au multiculturalisme. Groupe de comédiens, « De la dignité », Libération, 22 août 1996.

35.. "Pour qu'une constitution soit bonne, il faut qu'elle soit fondée sur les droits de l'homme, et qu'elle les protège évidemment; il faut donc pour préparer une constitution, connaître les droits que la justice naturelle accorde à tous les individus; il faut rappeler tous les principes qui doivent former la base de toute espèce de société, et que chaque article de la constitution puisse être la conséquence d'un principe. » Mounier, cité par P. Lavigne, Encyclopaedia universalis, vol. VI, 1984, p. 433.

36.. N. Rouland, « Trois ancrages de l'identité française », Libération, 3 avril 1995.

37. Cf. la position du Conseil d'État du 24 août 1996 sur les conditions d'application des lois sur l'immigration : "Il ne peut exister un droit à la régularisation, expression contradictoire en elle-même. La régularisation par définition est accordée dans l'hypothèse où le demandeur [...] ne bénéficie pas d'un droit, sinon il suffirait qu'il le fasse valoir », Libération, 25 août 1996. 
38.. " Pétition pour les sans-papiers ", signée par P. Bourdieu (sociologue), M. Augé, (anthropologue) A. Langaney (anthropobiologiste), etc., Libération, 18 septembre 1996. 39.. C. Pringent et F. Tanguy, « Qui creuse la fracture sociale ? ", Libération, 20-21 avril 1996.

40.. A. Touraine (sociologue), Qu'est-ce que la démocratie ?, Fayard, Paris, 1994.

41.. B. Ginisty, «Contre la pureté janséniste de l'économique », Libération, 11-12 décembre 1995.

42.. La perspective théorique du big-bang.

43. M. Wieviorka, « Histoire-mémoire, le lien nécessaire », Libération, 15 mars 1995.

44.. D. Bourcier, E. Catta, « Comment recoudre le droit », Libération, 26 juin 1995.

45.. J. Testart, B. Edelman (avocat), J. Friedman (juriste), N. Rouland (juriste).

46.. E. Pisani, « "Tous ensemble" contre la mondialisation », Le Monde diplomatique, janvier 1996.

47.. " La priorité politique ne doit plus être à l'insertion mais à la socialisation dans des activités ne constituant pas un secteur à part, mais une composante légitime de l'économie moderne relevant du droit commun ", P. Laville (sociologue), "Au-delà de l'insertion ", Libération, 15 mars 1995.

48.. J. Dupuy (sociologue et philosophe), «Égalité, équité, confusion », à propos du rapport Minc, Libération, 25 février 1995.

49.. En référence avec la Constitution de 1946 qui énonce les principes politiques, sociaux, économiques particulièrement nécessaires à notre temps.

50.. E. Morin (sociologue), Conférences de l'Atrium, juin 1994.

51.. En terme de patrimoine commun à l'humanité : droits biogénétiques, droits de l'enfant, droits de l'environnement...

\section{RÉSUMÉS}

A partir des commentaires, articles, témoignages et points de vue recueillis, d'octobre 1994 à décembre 1996, dans les quotidiens Libération et Le Monde, et dans le mensuel Le Monde diplomatique, l'auteur tente de cerner les formes et le sens de l'action collective au nom de l'exclusion.

\section{The banished : a legal minority or a community in fact}

From articles found in the newspapers Libération, Le Monde, and Le Monde diplomatique, the author makes an attempt to understand the forms and meaning of collective initiative against exclusion.

\section{INDEX}

Mots-clés : exclusion, identités, appartenance, intégration citoyenne, homme, droits fondamentaux de l'homme, communautés d'appartenance

Keywords : identity, community fellowship, fundamental human rights, citizenship integration 


\section{AUTEUR}

\section{BÉATRICE MESINI}

Béatrice MESINI est chargée de recherches au CNRS (UMR Telemme, Aix-en-Provence). Croisant les sources écrites, orales et audiovisuelles, elle s'attache à cerner comment le réel et la fiction composent le groupe des « exclus». 